\title{
HEAD TREMOR IN PATIENTS WITH CERVICAL DYSTONIA
}

\section{Different outcome?}

\author{
Clecio Godeiro-Junior, Andre C. Felicio, Patricia C. Aguiar, \\ Vanderci Borges, Sonia M.A. Silva, Henrique B. Ferraz
}

\begin{abstract}
Objective: The association of cervical dystonia (CD) with other movement disorders have been already described, but data on clinical outcome regarding these patients are scant. The aim of this paper was to investigate whether patients with $C D$ and head tremor $(\mathrm{HT})$ would have a different outcome regarding to botulinum toxin type-A (BTX-A) treatment response and clinical and demographic parameters. Method: We retrospectively evaluated 118 medical charts of patients with $C D$ and divided them into two groups: with $(\mathrm{HT}+)$ and without (HT-) head tremor. We compared the following clinical and demographic parameters: age at onset, disease duration, progression of symptoms, etiology, familial history, presence of hand tremor. We also analyzed the response to BTX-A according to Tsui score in both groups. Results: The occurrence of head tremor in our sample was of $38.2 \%$. The occurrence of postural hand tremor in the patients from the HT+ group was higher than in the HT- one $(\mathrm{p}=0.015)$ and if we compare BTX-A response in each group, we observe that patients with HT present a better outcome in a setting of longer follow-up. In HT+ group, Tsui score pre treatment was 10 (6-12.5) and after follow-up was 8 (5.5-10.5); $p<0.001$. In HT- group there was no significant difference 9 (7-12) in pre treatment and after follow-up; $p=0.07$. Conclusion: According to our data it seems that head tremor may influence the clinical outcome or treatment response with BTX-A in patients with CD.
\end{abstract}

KEY WORDS: cervical dystonia, head tremor, botulinum toxin type-A, outcome.

\section{Tremor cefálico em pacientes com distonia cervical: evolução diferente?}

Resumo - Objetivo: A associação de distonia cervical (DC) com outros transtornos do movimento já foi descrita, mas há poucos dados quanto à evolução clínica destes pacientes. Avaliamos se os pacientes com DC e tremor cefálico (TC) apresentam características clínicas e demográficas, assim como a resposta ao tratamento com toxina botulínica tipo A, diferentes. Métodos: Analisamos retrospectivamente 118 prontuários de pacientes com DC e os dividimos em dois grupos: com (TC+) e sem (TC-) tremor cefálico. Comparamos os seguintes parâmetros clínicos e demográficos entre os grupos: idade de início, duração da doença, progressão de sintomas, etiologia, história familiar, presença de tremor em mãos. Também analisamos a resposta ao tratamento com toxina botulínica de acordo com escore Tsui em ambos os grupos. Resultados: A ocorrência de tremor cefálico em nosso grupo de pacientes foi 38,2\%. A ocorrência de tremor postural nas mãos em pacientes TC+ foi maior $(p=0,015)$ e se compararmos a resposta ao tratamento com toxina botulínica em cada grupo, observamos que os pacientes com TC têm melhor evolução em uma situação de seguimento mais prolongado. No grupo TC+, o escore Tsui pré-tratamento foi 10 (6-12,5) e após o período de "follow-up" foi 8 (5,5-10,5); $p<0,001$. No grupo TC-, não houve diferença significante: 9 (7-12) no pré-tratamento e após período de "follow-up"; $p=0,07$. Conclusão: Nossos dados apontam que o tremor cefálico pode influenciar a evolução clínica ou resposta ao tratamento com toxina botulínica em pacientes com DC.

PALAVRAS-CHAVE: distonia cervical, tremor cefálico, toxina botulínica, evolução.

Cervical dystonia (CD) is a focal dystonia characterized by sustained, involuntary contractions of the neck muscles, resulting in abnormal movements and postures of the head'. It is often associated with other movement disorders, particularly tremor. Some consider that essen- tial tremor and idiopathic dystonia may be genetically linked ${ }^{2}$. The mechanism underlying $C D$ and head tremor is unknown, but its association is frequent as assessed with other studies ${ }^{3}$.

Our group previously reviewed 185 patients with idio-

Movement Disorders Unit, Department of Neurology and Neurosurgery, Federal University of São Paulo, São Paulo SP, Brazil.

Received 6 August 2008, received in final form 19 September 2008. Accepted 14 October 2008.

Dr. Clecio Godeiro-Junior - Rua Dr. Diogo de Faria 650 / 33 -04037-002 São Paulo SP - Brasil. E-mail: cleciojunior@yahoo.com.br 
pathic and symptomatic dystonia searching for the coexistence of postural hand tremor. The similar frequency of tremor in idiopathic and symptomatic dystonia patients suggests that the pathophysiologic derangement resulting in dystonia can favor the development of tremor ${ }^{4}$. However, we do not know how the coexistence of tremor in $C D$ patients, particularly in the head, may interfere in the natural history of their disease.

The purpose of this study was to compare demographic, clinical and botulinum toxin type-A (BTX-A) therapeutic response between two groups of $C D$ patients: with head tremor $(\mathrm{HT}+)$ and without head tremor $(\mathrm{HT}-)$.

\section{METHOD}

We retrospectively reviewed 184 charts of patients with CD who underwent medical treatment with BTX-A at the Movement Disorders Unit of our institution between 1990 and 2007. We included all patients who were submitted at least to one BTX-A application per year during follow-up, and excluded all patients with incomplete medical records or who's BTX-A injection interval exceeded 12 months.
We found 118 eligible patients and they were evaluated according to sex, age of $C D$ at onset, family history of $C D$, and Tsui scale score ${ }^{5}$ at first medical evaluation. Details of the dystonic movement were also reviewed: torticollis, anterocollis, retrocollis, laterocollis, head tremor, pain and concomitant postural hand tremor. The patients were divided according to presence of head tremor in their first evaluation into two groups: with head tremor $(\mathrm{HT}+)$ and without head tremor ( $\mathrm{HT}-)$.

According to the etiology of $C D$, we divided our patients into two groups: primary, when there was no evidence on history, examination or laboratory investigation of any identifiable cause of the dystonia; and secondary, when the cause of dystonia was identifiable such as anoxia, central or peripheral trauma, neuropathy, infection, metabolic disorder, or previous use of neuroleptic drugs. We also observed if the CD spreaded to another body parts. The pattern of progression included the following dystonic subtypes: blepharospasm, Meige syndrome, lower or upper limb dystonia, hemidystonia, multifocal or generalized dystonia. The clinical response to the treatment with BTX-A was assessed according to follow-up period, number of applications and Tsui score after follow-up.

Table 1. Demographic and clinical characteristics of patients with cervical dystonia.

\begin{tabular}{|c|c|c|c|}
\hline Groups & Head tremor $(+)$ & Head tremor (-) & $p$ value \\
\hline Number of patients enrolled & $\mathrm{n}=45$ & $\mathrm{n}=73$ & \\
\hline Men : Women & 1:1.2 & $1: 1.8$ & $0.06^{a}$ \\
\hline Age at onset (years) & $38 \pm 2.4^{*}$ & $39 \pm 1.8^{*}$ & $0.75^{\mathrm{b}}$ \\
\hline Disease duration (months) & $82 \pm 14 *$ & $59 \pm 9.4^{*}$ & $0.15^{\mathrm{b}}$ \\
\hline Familial history & $1(2.23 \%)$ & $9(12.3 \%)$ & $0.12^{\mathrm{c}}$ \\
\hline Primary : secondary & 2.5:1 & 2.3:1 & $0.95^{c}$ \\
\hline Progression (spreading) & $13(28.9 \%)$ & $33(45.2 \%)$ & $0.12^{c}$ \\
\hline Postural hand tremor & $11(24.5 \%)$ & $5(6.8 \%)$ & $0.015^{c}$ \\
\hline Pain & $36(80 \%)$ & $49(67.2 \%)$ & $0.2^{c}$ \\
\hline Torticollis & $6(13.4 \%)$ & $17(23.3 \%)$ & $0.27^{c}$ \\
\hline Laterocollis & $0(0 \%)$ & $1(1.4 \%)$ & $0.8^{\mathrm{c}}$ \\
\hline Anterocollis & $0(0 \%)$ & $3(4.1 \%)$ & $0.44^{c}$ \\
\hline Retrocollis & $2(4.4 \%)$ & $3(4.1 \%)$ & $0.71^{\mathrm{c}}$ \\
\hline Torticollis + laterocollis & $12(26.6 \%)$ & $17(23.3 \%)$ & $0.74^{c}$ \\
\hline Torticollis + anterocollis & $4(8.9 \%)$ & $5(6.8 \%)$ & $0.96^{c}$ \\
\hline Torticollis + retrocollis & $7(15.6 \%)$ & $14(19.2 \%)$ & $0.8^{\mathrm{c}}$ \\
\hline Torticollis + laterocollis + anterocollis & $9(20 \%)$ & $9(12.3 \%)$ & $0.38^{c}$ \\
\hline Torticollis + laterocollis + retrocollis & $5(11.1 \%)$ & $4(5.5 \%)$ & $0.44^{c}$ \\
\hline Tsui score pre treatment & $10(6-12.5)$ & $9(7-12)$ & $0.35^{\mathrm{d}}$ \\
\hline Tsui score post treatment (30 day) & $4(3-7)$ & $5(3-8)$ & $0.84^{d}$ \\
\hline Follow-up period (months) & $50(20-60)$ & $30(10-60)$ & $0.034^{d}$ \\
\hline Number of BTX-A applications & $5(3-8)$ & $4(2-5)$ & $0.13^{d}$ \\
\hline Tsui score pos treatment (follow-up) & $8(5.5-10.5)$ & $9(7-12)$ & $0.7^{d}$ \\
\hline
\end{tabular}

*Median and standard deviation; ${ }^{a}$ Chi-square test; ${ }^{b}$ Student $\mathrm{t}$ test; ${ }^{\mathrm{c} C h i-s q u a r e}$ with Yates correction; ${ }^{\mathrm{d}}$ Mann-Whitman test. BTX-A, botulinum toxin type-A. Significance $p<0.05$. 
Statistical analysis for continuous variables and comparisons between subgroups were performed by Student t-test (or MannWhitney, Wilcoxon signed rank, for non-parametric data); for categorical variables, comparisons between groups were performed using the Chi-square test, and, when necessary, with Yates correction. Significant values of $p$ were set at $\leq 0.05$. All statistical analyses were performed using software Prism 3.0. Parametric data are presented as mean \pm standard deviation (SD) and nonparametric data as median \pm percentile $\left(25^{\text {th }}\right.$ and $75^{\text {th }}$ percentiles).

This protocol was submitted to and approved by the ethics committee of our institution.

\section{RESULTS}

Among the 118 enrolled CD patients, 45 (38.2\%) presented HT in their first evaluation. We did not categorize HT as dystonic, essential or both. The demographic, clinical and therapeutic characteristics of the two groups (HT+ and $\mathrm{HT}^{-}$) are summarized on Table 1.

In the two groups we observed patients with $C D$ of either primary or secondary etiology. In the HT+ group there were $32(71.2 \%)$ patients with primary etiology and $13(28.8 \%)$ of secondary, while in the HT- group they were $51(69.8 \%)$ and $22(30.2 \%)$, respectively. In the HT+ group, the secondary etiologies were: use of neuroleptic $(n=7)$, cranial trauma $(n=3)$, anoxia $(n=2)$ and use of calcium channel blockers ( $n=1)$. In the HT- group, they were: use of neuroleptic ( $n=13$ ), meningitis ( $n=2$ ), cranial and cervical trauma $(n=2)$, anoxia $(n=1)$, Kernicterus $(n=1)$, multiple sclerosis $(n=1)$, atypical Parkinsonism ( $n=1)$, and Huntington disease $(n=1)$.

Clinical presentation was similar in both groups, except that we found a higher prevalence of postural hand tremor in the $\mathrm{HT}+$ group ( $24.5 \%$ vs. $6.8 \%, \mathrm{p}=0.015$ ) (Table 1). With regard to the progression of CD $28.9 \%$ patients of the $\mathrm{HT}+$ group and $45.2 \%$ of the $\mathrm{HT}$ - group spreaded to other body parts, but there was no statiscally significant difference.

Both groups received BTX-A in different presentations: Botox ${ }^{\circledR}$ or Dysport ${ }^{\circledR}$. Sixty-nine patients received Dysport ${ }^{\circledR}$ : in $\mathrm{HT}+$ group ( $\mathrm{n}=29$ ), the media dose was $513.8 \pm 142$ (standard deviation) and in the HT- $(n=40)$, it was $509 \pm 111$; $\mathrm{p}=0.8$. Forty-nine patients received Botox ${ }^{\circledR}$ : in $\mathrm{HT}+$ group $(n=16)$, the media dose was $273.8 \pm 76.5$ and in the HT-, 289.4 $\pm 64.4 ; p=0.76$. We did not use electromyography or amplifier during toxin injection. Despite the heterogeneity of both groups of patients with respect to their etiologies and BTX-A presentation, Tsui score pre treatment and clinical response to BTX-A were similar. We also compared Tsui score after follow-up period and found no difference, although follow-up of subjects from the HT+ group was longer than in the HT- group (Table 1). However, comparing Tsui score pre-treatment and after follow-up in each group, there was significant difference in $\mathrm{HT}+$ group (Table 2).

\section{DISCUSSION}

According to our data, patients with $\mathrm{CD}$ and $\mathrm{HT}+$ do not present different clinical findings from those without $\mathrm{HT}$-, except for higher number of patients with postural hand tremor in the former group (24.5\%).

There are some reports describing associations of tremor and $C D^{2,3,5-7}$. In a series of 114 patients with idiopathic CD it was found $68.4 \%$ with head tremor and $40 \%$ of them also had postural hand tremor that resembled essential tremor. Another large series ${ }^{2}$ reviewed 272 patients with $C D$ and complete information about tremor and found that $71 \%$ had tremor; $60 \%$ had associated headneck tremor, and $27 \%$ had hand tremor phenomenologically identical to essential tremor. A larger series ${ }^{6}$ showed a prevalence of head and hand tremor, respectively, of $50 \%$ and $32 \%$ in their 231 patients with CD, different from other report with similar sample that observed in 266 CD patients $28 \%$ had head tremor and $23 \%$ hand tremor ${ }^{7}$. Our data are similar with the aforementioned studies; we found in 118 patients with CD, that 45 (38.2\%) had head tremor and $24.5 \%$ of them also had postural hand tremor.

There was no difference of male-to-female ratio in both groups (1:1.2 vs. 1:1.8, HT+ vs. HT-, respectively). Another series demonstrated that there was a female predominance in the HT- group ${ }^{3}$. In general, female outnumber men at ratio 3:2 in large CD series ${ }^{8,9}$. Onset age did not differ between our groups and this finding is in line with literature data ${ }^{3}$.

We did not examine the family members and therefore data on family records may not be accurate. This could explain why we found only one case of family history in the HT+ group. A previous report ${ }^{2}$ showed that there was a higher frequency of dystonia and tremor in relatives of patients with dystonia. We found a higher prevalence of postural hand tremor in $\mathrm{HT}+$ group. The frequent occurrence of essential tremor in relatives of and in patients

Table 2. BTX-A treatment response in each group.

\begin{tabular}{lccc}
\hline Groups & Tsui score pre treatment & Tsui score pos treatment (Follow-up) & p value \\
\hline Head tremor (+) & $10(6-12.5)$ & $8(5.5-10.5)$ & $<0.001^{\mathrm{a}}$ \\
Head tremor (-) & $9(7-12)$ & $9(7-12)$ & $0.07^{\mathrm{a}}$ \\
\hline
\end{tabular}

All values are presented as median and $25^{\text {th }}-75^{\text {th }}$ percentiles. ${ }^{a}$ Wilcoxon signed rank test; significance $p<0.05$. 
with $C D$ may suggest a link between $C D$ and essential tremor as well as a role of genetic factors in the pathogenesis of $C D^{10}$.

Data on the clinical presentation of CD between HT+ and HT- groups did not differ. We would expect a higher occurrence of neck pain in the $\mathrm{HT}+$ group, once there is a higher association with head/neck trauma ${ }^{3}$, but in our patients there was no significant difference between groups ( $80 \%$ vs. $67.2 \%$, HT+ vs. HT- respectively). The pattern of CD may be of four types (torticollis, laterocollis, retrocollis or anterocollis) which may happen isolated (one type) or in combination (two or three types). Camargo et al. ${ }^{11}$ described a series with 85 patients with CD, and the most common clinical presentations were, according to the pattern: a) one type: torticollis (21 patients); b) two types: torticollis + retrocollis (19 patients); and c) three types: torticollis + laterocollis + retrocollis (09 patients). In our series, our results were different when the pattern of $C D$ involved two or three types: a) two types: torticollis + laterocollis (12 patients in HT+ group and 17 in HT-); and in b) three type: torticollis +laterocollis + anterocollis (9 patients in each group). This difference may be explained by the heterogeneity and larger number of patients in our sample.

We found secondary causes for CD in both groups with similar prevalence $(28.8 \%$ vs. $30.2 \%$, HT+ vs. HT- respectively). Unfortunately, due to multiple etiologies, these cases do not provide any clues to the understanding of $C D$ pathogenesis. Other interesting observation is that in both groups ( $\mathrm{HT}+$ and $\mathrm{HT}-$ ) some patients spreaded dystonia to other body parts.

The advent of BTX-A injections changed the natural course of $C D^{12}$. In our sample of patients there was significant difference between follow-up of $\mathrm{HT}+$ (50 months) and HT- (30 months). We also noted that there was no significant difference between the number of BTX-A injections ( 5 vs. 4 , respectively on $\mathrm{HT}+$ and $\mathrm{HT}-$ ) during this follow-up. Comparing Tsui score 30-days after the first application of BTX-A and after follow-up period, we could infer that there was no clinical difference between groups. However, if we compare Tsui score pre-treatment and after follow-up in each group, there is a significant difference in the HT+ group (Table 2), while in the HT- Tsui score remains similar. We may infer that BTX-A is effective in both groups, but in a long term basis, those patients with $\mathrm{HT}+$ present a better response once their symptoms tend to ameliorate. Our data is different with another report ${ }^{3}$ which demonstrated that the response to BTX-A was also as effective in patients with HT as in those without, suggesting that the presence of head tremor itself was not a predictor of poor outcome ${ }^{3}$. In our series, probably due to longer follow-up, we observed that patients with HT present a better outcome.

Our study presented some limitations, especially due to its retrospective nature. Data collected was based on information obtained from medical charts with the participation of different physicians throughout the period of this study. However, the final diagnosis and Tsui scale score were made by a full trained movement disorder specialist (HBF, VB, SMAS, and PMCA). Moreover, BTX-A injection doses were not standard among patients treated and we could not either categorize the pattern of tremor (dystonic, essential or combination) or identify it in other body regions, for example trunk and voice. Additionally, antibody testing was not carried out and we did not register adverse events to BTX-A therapy.

In conclusion, $\mathrm{CD}$ patients with $\mathrm{HT}$ have similar clinical patterns and BTX-A response to those without. There is a higher incidence of postural hand tremor; besides, if we compare BTX-A response in each group, we observe that patients with HT present a better outcome in a setting of longer follow-up.

\section{REFERENCES}

1. Fahn S. Concept and classifications of dystonia. Adv Neurol 1988;50:1-8.

2. Jankovic J, Leder S, Warner D, Schwartz K. Cervical dystonia: clinical findings and associated movement disorders. Neurology 1991;41:1088-1091.

3. Pal PK, Samii A, Schulzer M, Mak E, Tsui JKC. Head tremor in crevical dystonia. Can J Neurol Sci 2000;27:137-142.

4. Ferraz HB, Andrade LAF, Silva SMA, Borges V, Rocha MSG. Tremor postural e distonia. Arq Neuropsiquiatr 1994;52:466-470.

5. Tsui JKC, Eisen A, Stoessel AJ, Calne S, Calne DB. Double-blind study of botulinum toxin in spasmodic torticolis. Lancet 1986;2:245-247.

6. Duane DD, Clark M, Gottlob L, Case JL, LaPointe LL. The influence of family history on cervical dystonia. Mov Disord 1993;8:413-414.

7. Chan J, Mitchell FB, Fahn S. Idiopathic cervical dystonia: clinical characteristics. Mov Disord 1991;5:119-126.

8. Lowenstein DH, Aminoff MJ. The clinical course of spasmodic torticollis. Neurology 1988;38:530-532

9. Jahanshahi M, Marion MH, Marsden D. Natural history of adult-onset idiopathic torticollis. Arch Neurol 1990;47:548-552.

10. Lou J, Jankovic. Essential tremor: clinical correlates in 350 patients. Neurology 1991;41:234-238.

11. Camargo CH, Teive HA, Becker N, Baran MH, Scola RH, Werneck LC. Cervical dystonia: clinical and therapeutic features in 85 patients. Arq Neuropsiquiatr 2008;66:15-21.

12. Skogseid IM, Kerty E. The course of cervical dystonia and patient satisfaction with long-term Botulinum toxin A treatment. Eur J Neurol 2005;12:163-170. 